\title{
Hacia una formación común en Bibliotecología: el caso colombiano
}

\author{
Towards a Common Academic Training in \\ Librarianship: The Colombian Case
}

\author{
Ruth Helena Vallejo Sierra* \\ Investigadora independiente
}

Recibido: 11 de marzo 2016 Aceptado: 03 de agosto 2016

Corregido: 11 de setiembre 2016 Publicado: 30 de noviembre 2016

\begin{abstract}
Resumen
Este artículo analiza la formación en bibliotecología como parte de la investigación La bibliotecología como profesión en Colombia, presentando el análisis comparativo de la formación en Colombia frente al título de Información y Documentación español; señalando sus puntos de encuentro y sus divergencias; intentando mostrar las principales áreas en que podrían darse acuerdos para establecer contenidos comunes obligatorios que debería tener cualquier programa de formación bibliotecológica en Colombia.
\end{abstract}

Palabras clave: Formación profesional, bibliotecología, currículo, formación profesional, perfil profesional.

\begin{abstract}
This article analyzes the librarianship academic formation, as part of the investigation Librarianship as a profession in Colombia, presenting a comparative analysis of training in Colombia with the Spanish Information and Documentation formation; indicating their common points and their differences; trying to show the main areas in which it could be agreement. In order to establish the common content that should be the obligatory core curriculum for any librarianship program in Colombia.
\end{abstract}

Keywords: Librarianship, professional studies, Curriculum, Professional Formation, Professional profile

\section{bibliotecas}




\section{Introducción}

Históricamente, el tema de la formación del bibliotecólogo cuenta con distintas miradas y posiciones. Shera (1990) presenta una división en tres periodos: el de aprendizaje y capacitación, que fue el servicio que prevaleció desde los tiempos antiguos hasta 1887; el de formación escolarizada bibliotecaria organizada, que va de 1887 a 1923, cuando apareció el informe Williamson; y finalmente la etapa de desarrollo de la escuela de bibliotecarios con enfoque académico.

Rodríguez (2001), partiendo de la necesidad de formar un bibliotecario que responda a las nuevas demandas de la sociedad, hace una caracterización de sus transformaciones en el tiempo: en los primeros años estaban encaminados a formar una cultura enciclopedista. El acceso era restringido, duraba más de diez años y su perfil era de eruditos, lo cual les permitía ser reconocidos y altamente apreciados por la sociedad.

La existencia de diversas propuestas de formación técnica, profesional y posgradual, con énfasis en las áreas de bibliotecología, archivística y documentación - unidas o en forma separada- y la aparición de la ciencia de la información son temas de debate continuo, con dos tendencias básicas: una en un saber único con la bibliotecología y en otra considerarla autónoma e independiente. Esta situación que no es ajena a las escuelas de bibliotecología en Latinoamérica, las cuales se han inscrito en una u otra concepción.

El balance sobre cómo se dio la formación en América Latina es considerado por Radamés Linares (2004) en dos partes: hacia los años cuarenta, con un especial énfasis en el dominio de técnicas para organizar y conservar fondos documentales, dando lugar a una carencia en los aspectos teóricos de la profesión. Posteriormente, en los años setenta, se incorporaron los primeros conocimientos tecnológicos que, así como en algunos casos, representaron un fortalecimiento de la profesión; en muchos otros se inició simultáneamente la discusión sobre el sentido o no de la profesión, dado que se centraban, especialmente, 
en los contenidos prácticos de organización y recuperación de la información, algunos contenidos de gestión y tecnologías y, en algunos casos, la formación general en idiomas y comunicación, entre otras áreas. Era evidente que aún no estaba clara la formación investigativa dentro de los currículos, como ya lo detectaba la Unesco en su encuesta de formación universitaria en escuelas de bibliotecología.

A partir de la gran cantidad de autores e instituciones que han escrito sobre el tema, es posible concluir, en general lo siguiente: 1) No se ha logrado unificar una oferta de contenidos, al punto de que continúa la discusión sobre el nivel de teoría y práctica que debe llevar cada proceso formativo. 2) Cada escuela se esfuerza por darle una ventaja competitiva a su plan de estudios, priorizando unos temas sobre otros y buscando hacer énfasis en la definición de sus perfiles sociales, tecnológicos o técnicos (como ocurre en algunos países centroamericanos), universitarios (apuesta que comparten la gran mayoría de los países iberoamericanos), a nivel de maestría (caso de los países anglosajones), incluyendo la acreditación de calidad, según las características definidas por la American Library Association.

De esta manera, tal como lo afirma Vallejo (2011), existen diversidad de currículos y titulaciones en toda Latinoamérica y experiencias de acuerdos como el de Mercosur; sin embargo, es indispensables contar con la voluntad política para lograr unos mínimos comunes en un perfil del profesional de la información latinoamericano que permita realmente dar avances significativos en el desarrollo de la profesión. Aunque hay que tener en cuenta como lo indica Ríos (2010) que esto implicaría algo más que lograr acuerdos y consensos; pues involucra un conjunto de reformas sobre los sistemas educativos de bibliotecología en el marco de un Espacio Latinoamericano de Educación Superior.

\section{La formación profesional en Colombia}

En cuanto a los primeros pasos recorridos para profesionalizar la formación se debe destacar el primer curso de bibliotecología realizado

\section{bibliotecas}


por Daniel Samper Ortega, director de la Biblioteca Nacional en la década de 1930. En 1942 se creó un programa regular de escuela de bibliotecarios en la Biblioteca Nacional, bajo los auspicios de ALA y con la financiación de la Fundación Rockefeller. En 1946 se fundó en Bogotá la primera Escuela de Bibliotecarios en el Colegio Mayor de Cundinamarca, por parte del Ministerio de Educación Nacional. Esta escuela finalizó sus labores en 1948. En 1956 el Fondo Universitario Nacional estudió la posibilidad de establecer en Bogotá una escuela para formar bibliotecarios universitarios. En Medellín se creó la Escuela de Biblioteconomía del Colegio Mayor de Antioquia (Universidad Femenina) en 1946. En 1964 el Banco de la República comenzó a dictar cursos de bibliotecología, los cuales continuaron hasta 1978.

Posteriormente, en consecuencia con el desarrollo de las bibliotecas universitarias y especializadas surgió la necesidad de multiplicar la capacitación de los bibliotecarios para que pudiesen incorporar técnicas desarrolladas en otros países, especialmente los anglosajones. En la década de los cincuenta del siglo xx el doctor Gaston Litton desarrolló cursos de formación para bibliotecarios en la Universidad Nacional, el Ministerio de Educación Nacional, el Departamento del Servicio Civil, la Biblioteca Nacional y la Biblioteca Luis Ángel Arango. Dicha capacitación se extendió a auxiliares de bibliotecas universitarias en Medellín.

Este avance inicial, al igual que los avances hechos a nivel internacional, empezó por fuera de las universidades, tratándose de cursos cortos y enfocados al manejo técnico de las bibliotecas. Por supuesto, el estatus del bibliotecario siempre se mantuvo en niveles bajos, así como sus condiciones salariales, pero ello contribuyó a forjar el camino para la materialización de iniciativas institucionales que condujeron a su capacitación.

Con este panorama podemos establecer la creación de una serie de instituciones de formación a nivel del país: la Escuela de Bibliotecarios de la Biblioteca Nacional en 1942; la Escuela de Biblioteconomía del Colegio Mayor de Cundinamarca en 1946, que dictó programas para bibliotecarios hasta 1954; la Escuela de Bibliotecología del Colegio Mayor de Cultura Femenina de Antioquia, con un programa de un año 
de duración ofrecido entre 1946 y 1959, y la Escuela de Bibliotecología del Colegio Académico de Antioquia, que en 1960 abrió la Escuela de Humanidades y Bibliotecología, la cual funcionó hasta 1967.

También es necesario mencionar a la Escuela de Bibliotecología y Archivística de la Universidad Nacional en Bogotá, que en 1965 creó la carrera de Bibliotecología y Archivística en la Facultad de Filosofía y Letras, programa que quedó en la sola iniciativa; la Escuela de Bibliotecarios del Colegio Universitario del Sagrado Corazón en Cali en 1967, con el programa de Tecnología en Bibliotecología, de seis semestres de duración, con la posibilidad de optar por la licenciatura con un año más en la Escuela Interamericana de Bibliotecología; y el programa de Formación de Bibliotecarios Escolares del Departamento de Bibliotecología y Recursos Educativos de la Universidad Pedagógica Nacional de Bogotá, creado en 1974 para formar en tres semestres expertos en bibliotecas escolares.

Iniciativas todas estas que prepararon el terreno para que posteriormente los bibliotecarios optaran por la profesionalización de su práctica, matriculándose en los programas ofrecidos en Bogotá, Medellín y Armenia. Así, nació en 1956 la Escuela Interamericana de Bibliotecología de Medellín, con Gaston Litton a la cabeza de sus fundadores, mientras que el programa de la Universidad de La Salle en Bogotá nació bajo la tutela del profesor León Jaime Zapata, quien con la influencia decisiva de fray Alberto Lee, director del Archivo Nacional, creó la primera escuela de bibliotecarios y archivistas en 1971. Dos años más tarde la Pontificia Universidad Javeriana de Bogotá inició la carrera de Bibliotecología y en 1987 la Universidad del Quindío abrió el programa en la modalidad virtual. En el 2011 apareció el Programa Profesional en Ciencia de la Información-Bibliotecología en INPAHU.

En cuanto a los programas de posgrado, en 1998 se estableció la Especialización en Redes de Información Documental en la Universidad Javeriana. En el 2001 se creó la Especialización en Servicios de Información de la Escuela Interamericana de Bibliotecología de la Universidad de Antioquia. Y en el 2011 aparecieron la Maestría en Ciencia de la In-

\section{bibliotecas}


formación de la Universidad de Antioquia y la Maestría en Gestión Documental y Administración de Archivos de la Universidad de La Salle.

Es de señalar que la formación técnica y tecnológica se vio impulsada en el 2001 con la aparición del programa de Tecnología en Administración de Sistemas de Información y Documentación del INPAHU y en el 2006 con el de Técnico en Bibliotecas del Instituto Técnico Abolsure (Cartagena). Los cursos de formación para el trabajo también se vieron favorecidos con la aparición del programa de Tecnología en Gestión de Bibliotecas del Servicio Nacional de Aprendizaje (Sena) en el 2010.

\section{a. Escuelas de formación profesional}

En el caso colombiano cualquier propuesta de educación superior debe estar comprendida dentro del marco de la Ley 30 de 1992, por la cual se organiza el servicio público de la Educación Superior y se establecen los niveles de formación reconocidos por el estado colombiano como se observa en la Tabla 1.

De acuerdo con los niveles de educación superior, a nivel tecnológico se encuentra el programa ofrecido por el INPAHU, con una duración de siete semestres. En el campo universitario se ha avanzado mucho en la profesionalización de la carrera, pues hoy el país cuenta con escuelas de bibliotecología en las universidades de Antioquia y su Escuela Interamericana de Bibliotecología, La Salle, Pontificia Universidad Javeriana, Quindío, ubicadas en Bogotá, Medellín y Armenia (virtual). Las tres primeras cuentan con acreditación como programas de alta calidad. En todas se otorga el título de bibliotecólogo, en cumplimiento de la Ley 11 de 1979, pero se han agregado otros alcances tales como profesional en ciencia de la información y profesional en sistemas de información. A partir del 2011 el INPAHU inició también su programa profesional.

En relación con los enfoques de formación en Colombia, esto sigue siendo una preocupación de muchos sectores en su afán por dar respuesta adecuada al desarrollo que reclama la profesión, teniendo en cuenta para ello los referentes del entorno, las necesidades del mercado y la impronta que 
Tabla 1

Niveles de formación en Educación Superior

\begin{tabular}{|c|c|c|c|}
\hline EDUCACIÓN SUPERIOR & TÉCNICO PROFESIONAL & TECNOLOGO PROFESIONAL & PROFESIONAL UNIVERSITARIO \\
\hline \multirow{2}{*}{ Créditos Académicos } & Entre 60-75 & Entre $90-110$ & Entre 150 y 170 \\
\hline & 4-5Semestres & 5-7 semestres & 8-12 semestres \\
\hline \multirow[t]{2}{*}{ Requisitos } & $\begin{array}{c}\text { Noveno grado grado culminado o } \\
\text { Título de Bachiller }\end{array}$ & Título de Bachiller & Título de Bachiller \\
\hline & Prueba de ICFES & Prueba de ICFES & Prueba de ICFES \\
\hline $\begin{array}{l}\text { Competencias que } \\
\text { adquiere }\end{array}$ & $\begin{array}{l}\text { Conocimientos teóricos específicos y } \\
\text { prácticos altamente calificados sobre } \\
\text { procesos de sectores de la producción }\end{array}$ & $\begin{array}{c}\text { Conocimientos teóricos generales y } \\
\text { científicos y conocimientos prácticos } \\
\text { sobre procesos de sectores de la } \\
\text { producción }\end{array}$ & $\begin{array}{c}\text { Conocimientos teóricos } \\
\text { fundamentados, científicos y } \\
\text { técnicos sobre un área específica del } \\
\text { saber o una disciplina }\end{array}$ \\
\hline Perfil & $\begin{array}{c}\text { Esta capacitado para trabajos que } \\
\text { requieran conocimientos técnicos y } \\
\text { competencias en áreas específicias de } \\
\text { los sectores de la producción }\end{array}$ & $\begin{array}{l}\text { Posee conocimientos técnicos y } \\
\text { fundamentación científica de un } \\
\text { oficio; tiene capacidad de } \\
\text { innovación, decisión y gestión }\end{array}$ & $\begin{array}{l}\text { Cuenta con fundamentación teóricay } \\
\text { metodológica de una profesión y } \\
\text { amplia formación para la dirección, el } \\
\text { diseño y la gestión }\end{array}$ \\
\hline Hacia donde ir después... & $\begin{array}{l}\text { Mercado laboral con título de técnico } \\
\text { profesional en..... Continuar su } \\
\text { formación como tecnólogo o como } \\
\text { profesional universitario (puede } \\
\text { requerir pruebas de suficiencia de } \\
\text { conocimientos) Especializarse }\end{array}$ & $\begin{array}{c}\text { Mercado laboral con título de } \\
\text { tecnólogo en..... Continuar su } \\
\text { formación como profesional } \\
\text { universitario (puede requerir pruebas } \\
\text { de suficiencia de conocimientos) } \\
\text { Especializarse }\end{array}$ & $\begin{array}{l}\text { Mercado laboral con título de } \\
\text { profesional universitario según área } \\
\text { de estudio. Especializarse }\end{array}$ \\
\hline
\end{tabular}

Fuente: elaboración propia. Basado en la Ley 30 de 1990 e información del Ministerio de Educación Nacional. ICFES se refiere al Instituto Colombiano de Educación Superior que realiza los exámenes de estado (pruebas saber) a los estudiantes de último año de formación secundaria.

cada escuela le imprime a sus "profesionales de la información”, con el fin de responder a una sociedad que demanda de ellos, su ejercicio competente con perfiles adecuados para desempeñarse en unidades de información, servicios, redes y sistemas relacionados con bibliotecas y archivos.

En este sentido, y desde las posturas educativas, se han planteado diferentes rutas formativas que intentan responder a factores que marcan la tendencia que guiará la apuesta formativa en una u otra o ambas disciplinas: bibliotecología y archivística, ante lo cual la Unesco (1987) sugiere que debe darse de manera armonizada, entendiendo que

\section{bibliotecas}


Armonizar no significa una capacitación idéntica para todos; se trata solamente de buscar los aspectos comunes de las diversas actividades profesionales a fin de facilitar los intercambios intelectuales, racionalizar la utilización de los recursos pedagógicos, organizar mejor el trabajo profesional e incluso, de ser posible, consolidar el peso de estas profesiones ante los dirigentes o la sociedad en general. Es evidente que deben mantenerse las identidades propias de las profesiones y, si la aplicación de un enfoque armonizado llevara a confundirlas, habría que abandonarlo de inmediato. (p. 2)

Este punto es tal vez donde se muestra la mayor polémica y diferenciación en los currículos colombianos, donde se presenta la integración, el énfasis o la independencia de cada profesión.

La integración se caracteriza por apuestas que sugieren una fusión de las disciplinas, partiendo del concepto del estudio de la información. De esta manera, cada espacio académico se analiza desde esta perspectiva asumiendo que la organización, el comportamiento y la recuperación de la información tienen que ser vistos de manera global, sin que se evidencien, más allá de los microcurrículos, las especificidades que pueda requerir cada disciplina.

Por su parte, los currículos con énfasis específicos en una u otra disciplina, mantienen espacios comunes que pueden ser abordados por ambas disciplinas de manera similar. En este caso, se presentan también espacios que como la organización de la información se plantea de manera totalmente independiente, pues es uno de los puntos centrales de su diferenciación, entendiendo que no es lo mismo la descripción bibliográfica que la descripción archivística o la gestión de colecciones que la gestión documental. 


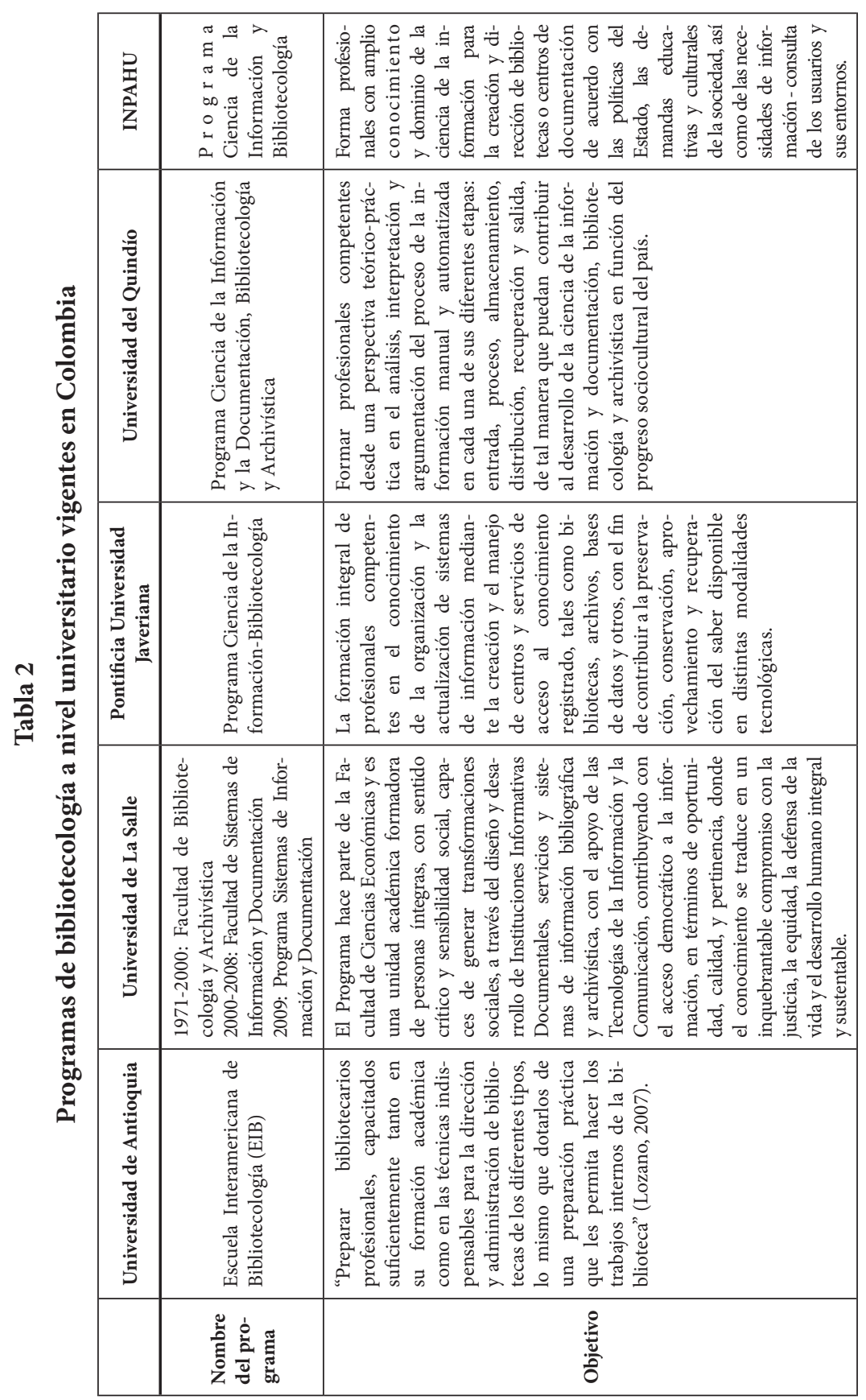

\section{bibliotecas}




\begin{tabular}{|c|c|c|}
\hline $\begin{array}{l}\text { 䒘 } \\
\text { 畜 }\end{array}$ & 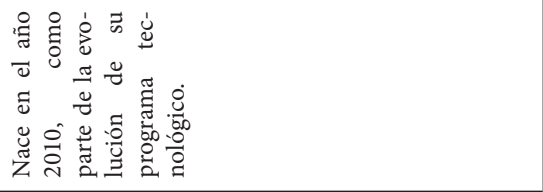 & \\
\hline 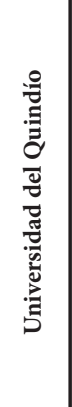 & 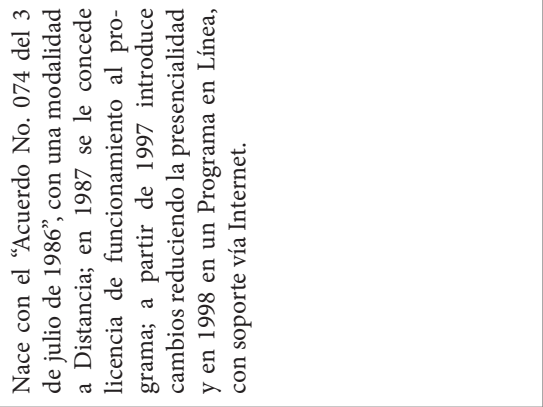 & 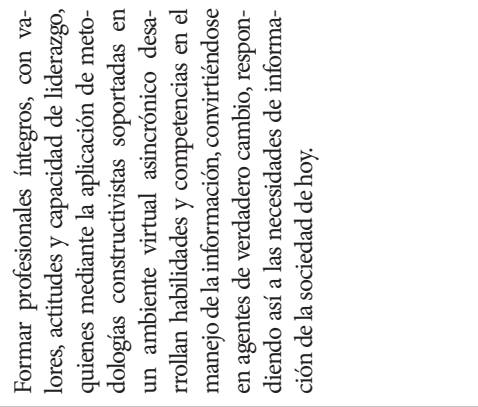 \\
\hline 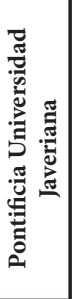 & 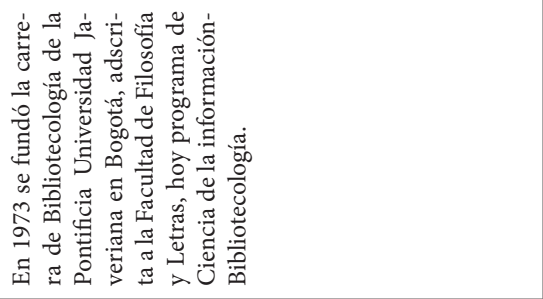 & \\
\hline 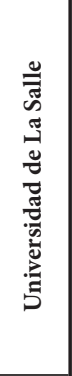 & 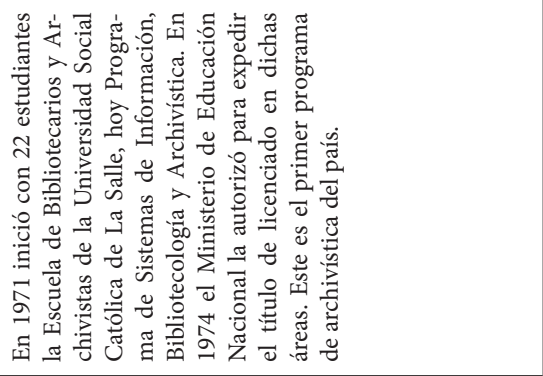 & 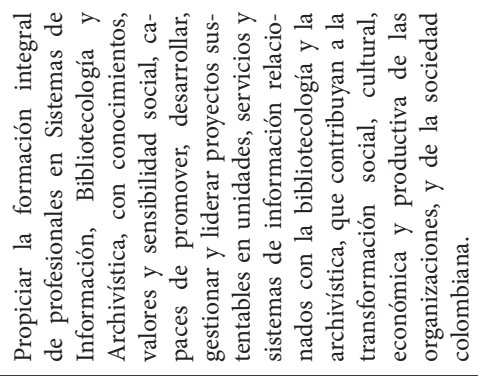 \\
\hline \multirow[t]{2}{*}{ 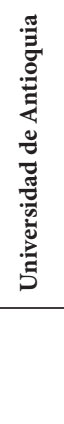 } & 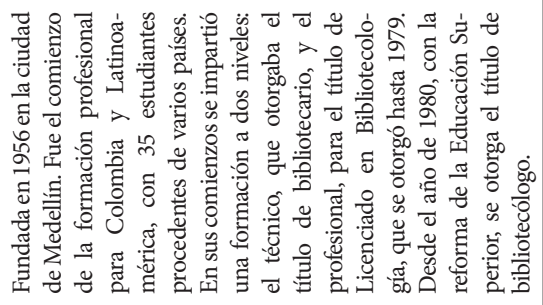 & 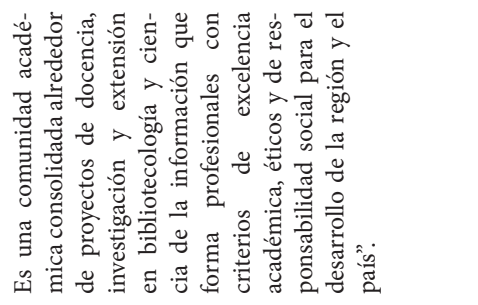 \\
\hline & 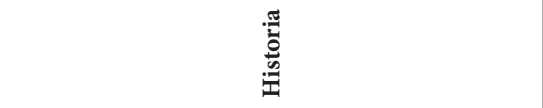 & 芯 \\
\hline
\end{tabular}


URL: http://www.revistas.una.ac.cr/index.php/bibliotecas/index

\begin{tabular}{|c|c|c|c|}
\hline $\begin{array}{l}\text { 胥 } \\
\text { 至 }\end{array}$ & & & 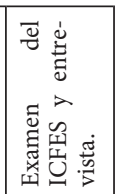 \\
\hline 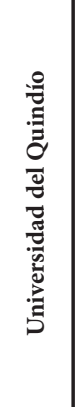 & 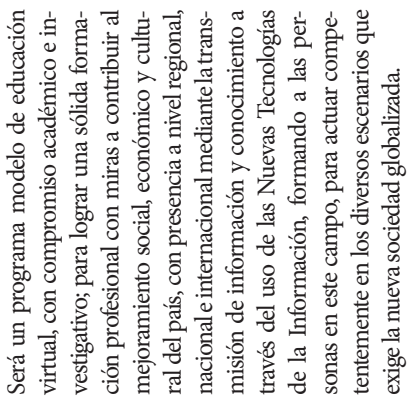 & 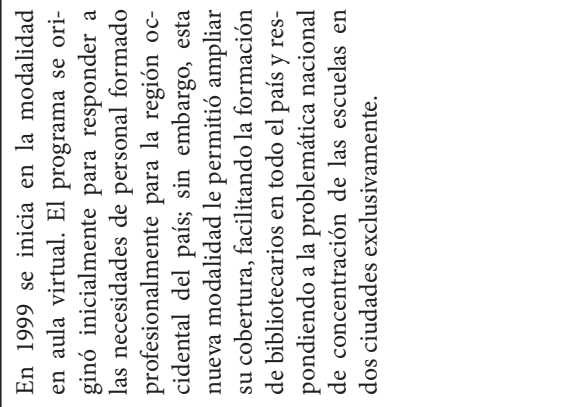 & 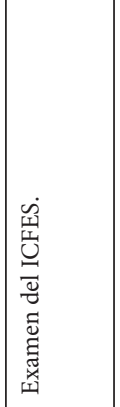 \\
\hline 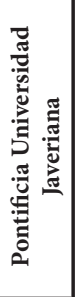 & & & 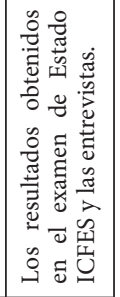 \\
\hline 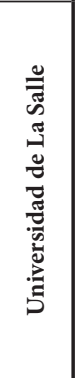 & 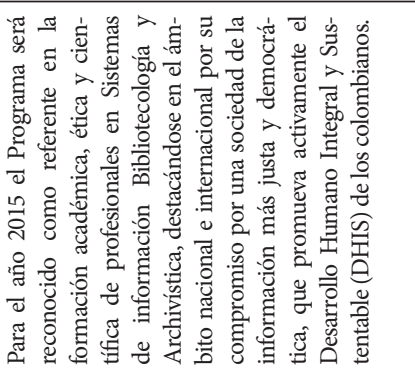 & 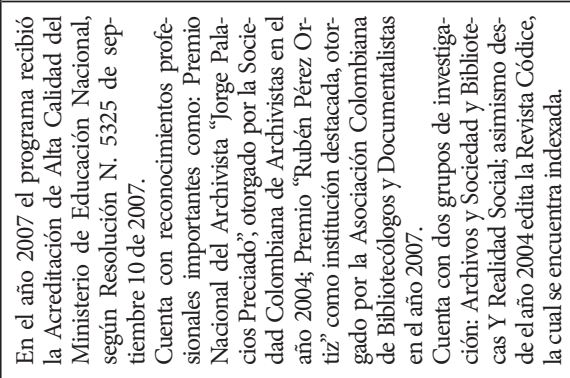 & 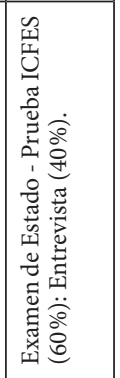 \\
\hline 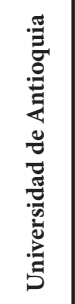 & 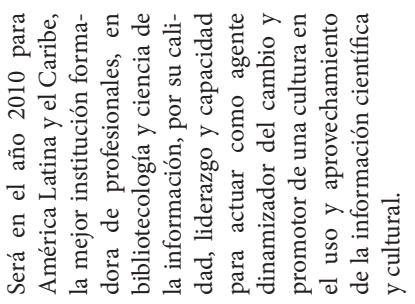 & 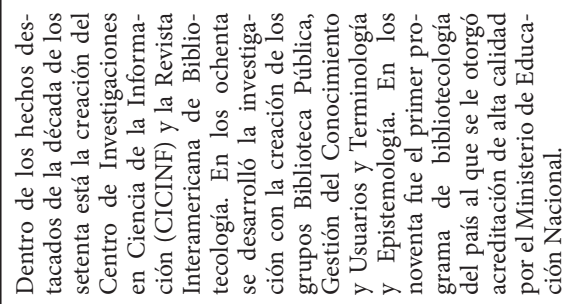 & 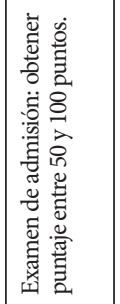 \\
\hline & 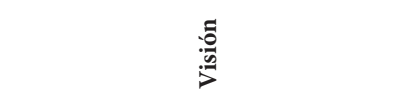 & 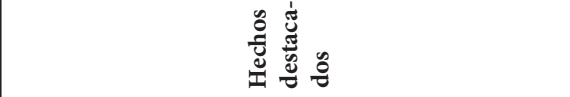 & 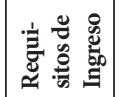 \\
\hline
\end{tabular}


En el caso de la independencia de cada profesión, la característica está dada por propuestas de planes de estudio independientes que pueden de alguna manera homologar espacios académicos. Estas concepciones, que deberían debatirse, precisarse y concretarse en discusiones teóricas de la profesión, generan hechos como:

- Titulaciones que otorgan los programas académicos están determinadas por el tipo de currículo. Se ofrece una gama de estas, así: a) profesional en ciencia de la información, bibliotecólogo, archivista; b) profesional de la información y c) profesional en sistemas de información. Ello contribuye a desdibujar la identidad profesional y confunde al mercado laboral que debería ser ajeno a estas discusiones que causan confusión a la hora de contratar profesionales en el área.

- Dificultad para desarrollar programas de intercambio, movilidad y homologación académica entre las diferentes instituciones.

- Inconvenientes en lograr una impronta que caracterice a un profesional según el programa de donde salga. Esto ha llevado a que se difumine la troncalidad de la profesión, al considerarse que ello se logra dándole mayor peso a áreas administrativas o tecnológicas, en detrimento de las áreas fundamentadoras de la profesión.-

- Puntos de encuentro de investigaciones, muchas veces difusos, pues la mirada desde cada programa dificulta apuestas comunes. Tal vez una discusión centrada en la epistemología y el desarrollo teórico pueda en el futuro aclarar estas divergencias.

- Exámenes de Estado para las profesiones han contribuido a tener que sentar las diferentes concepciones en torno a la pregunta: ¿cómo construir una base sobre la cual evaluar los conocimientos mínimos que un profesional de ésta área debe poseer?

De acuerdo con las condiciones de Colombia, se puede resumir la formación profesional así:

- Existen cinco escuelas de formación profesional, cada una con una tendencia diferente. 
- El Estado reconoce de manera independiente el ejercicio profesional del bibliotecólogo (Ley 11 de 1979) y del archivista (Ley 1409 de 2010).

- Actualmente se realizan debates por la expedición de tarjetas profesionales independientes: una de bibliotecólogo y la otra de archivista, a partir de una sola titulación.

- Las titulaciones profesionales son diversas. Por exigencia de la ley, como palabra común a todas ellas se encuentra bibliotecólogo y, en las que así lo han definido, también archivista

- El estado colombiano ha establecido que los profesionales de todas las áreas del conocimiento deben presentar pruebas de saber. Para ello en una primera fase de implementación se ha exigido el examen de pruebas básicas que miden competencias argumentativas, escriturales, lectoras etc., y, paralelamente, se va implementando la evaluación de competencias específicas por áreas del conocimiento. La discusión ahora se centra en si la profesión debe presentar exámenes específicos y qué pasaría en estos exámenes habida cuenta de la diversidad de la formación.

Esta contextualización nos permite identificar las diferencias formativas que existen en el país, como se presenta en la Tabla 3.

Tabla 3

Escuelas de Formación Profesional en Colombia

\begin{tabular}{lll}
\hline \multicolumn{1}{c}{ UNIVERSIDAD } & \multicolumn{1}{c}{ PROGRAMA } & \multicolumn{1}{c}{ Formación } \\
\hline Universidad de Antioquia & Escuela Interamericana de Bibliotecología & Independiente \\
\hline Universidad de La Salle & Sistemas de Información y Documentación & Énfasis \\
\hline Universidad Javeriana & Ciencias de La Información- Bibliotecología & Integración \\
\hline INPAHU & Ciencias de la Información-Bibliotecología & Énfasis \\
\hline Universidad del Quindío & $\begin{array}{l}\text { Ciencia de la Información y la Documenta- } \\
\text { ción, Bibliotecología y Archivística }\end{array}$ & Énfasis \\
\hline
\end{tabular}

Fuente: elaboración propia.

\section{bibliotecas}




\section{Metodología}

Para comprender mejor los procesos formativos que se llevan a cabo en el país, se parte de los planes de estudio de acuerdo con las páginas web de todas las escuelas de bibliotecología y se comparara con los contenidos comunes obligatorios del título de grado en Información y Documentación español, de manera que se puedan observar los puntos nucleares, las diferencias generales y los matices que cada escuela ofrece como valor agregado (ver Tabla 4).

\section{Tabla 4}

Propuesta de contenidos comunes obligatorios (troncalidad) del título de grado en Información y Documentación

\section{Documentos, unidades y sistemas de información. 12 créditos.}

Naturaleza de la información. Concepto y evolución de los documentos. Naturaleza y evolución de los archivos, las bibliotecas y los centros y servicios de documentación. Desarrollo histórico y estado actual de la disciplina. Legislación y políticas nacionales e internacionales. Aspectos culturales, sociales, económicos y legales de la producción y el uso de la información documental. Deontología y perfil del profesional de la Información y Documentación.

Competencias:

Interacción con los usuarios y clientes de la información (nivel 1).

Conocimiento del entorno profesional de la información y la documentación (nivel 3).

Conocimiento del marco jurídico y administrativo de la gestión de la información (nivel 2).

2. Planificación, organización y evaluación de unidades de información. 24 créditos. Planificación, organización y evaluación de recursos, procesos, servicios y productos de información. Desarrollo y mantenimiento de fondos y colecciones. Programas de conservación y preservación de documentos. Técnicas de gestión de recursos humanos y financieros. Técnicas de promoción: marketing y formación de usuarios.

Competencias:

Interacción con los usuarios y clientes de la información (nivel 2).

Gestión de colecciones y fondos (2).

Conservación y tratamiento físico de documentos (nivel 1).

Técnicas de gestión administrativa (nivel 2).

Técnicas de marketing (nivel 2).

Técnicas comerciales (nivel 1).

Técnicas de adquisición (nivel 1).

Técnicas de gestión microeconómica (nivel 2).

Técnicas de instalación, acondicionamiento y equipamiento (nivel 2).

Técnicas de planificación y gestión de proyectos (nivel 2).

Técnicas de diagnóstico y evaluación (nivel 2).

Técnicas de gestión de recursos humanos (nivel 2).

Técnicas de formación (nivel 1). 


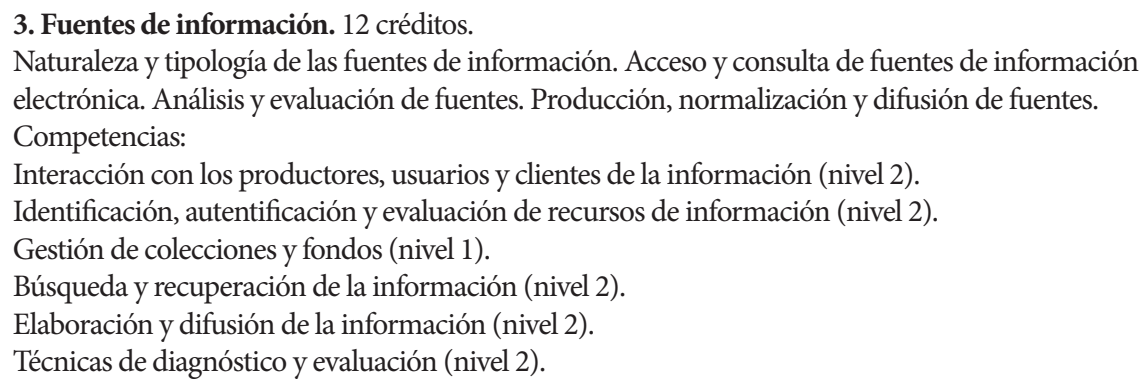

\section{Representación y recuperación de la información. 36 créditos.}

Catalogación descriptiva. Indización. Clasificación. Resumen. Desarrollo, mantenimiento y evaluación de instrumentos de organización, control y acceso. Elaboración, mantenimiento y uso de lenguajes documentales. Técnicas de recuperación de información. Sistemas avanzados de recuperación de información. Evaluación de la recuperación de información.

Competencias:

Análisis y representación de la información (nivel 2).

Organización y almacenamiento de la información (nivel 2).

Búsqueda y recuperación de la información (nivel 2).

Elaboración y difusión de la información (nivel 2).

Tecnologías de la información: Informática (nivel 2).

Técnicas de diagnóstico y evaluación (nivel 2).

\section{Gestión técnica de documentos de archivo. 18 créditos.}

Organización de fondos de archivo. Identificación y valoración. Clasificación y descripción.

Acceso y difusión. Gestión del ciclo de vida de los documentos administrativos.

Competencias:

Identificación, autentificación y evaluación de recursos de información (nivel 2).

Gestión de colecciones y fondos (nivel 2).

Conservación y tratamiento físico de los documentos (nivel 2).

Análisis y representación de la información (nivel 2).

Organización y almacenamiento de la información (nivel 2).

Búsqueda y recuperación de la información (nivel 2).

Elaboración y difusión de la información (nivel 2).

\section{Tecnologías de la información y edición digital. 24 créditos.}

Arquitectura de ordenadores. Sistemas operativos. Lenguajes de ordenadores. Sistemas y redes de comunicación. Arquitectura de la información. Programas de aplicación documental. Fundamentos y diseño de bases de datos. Edición de documentos digitales. Bibliotecas digitales.

Competencias:

Tecnologías de la información: Informática (nivel 2).

Tecnologías de la información: Redes (nivel 2).

Técnicas de producción y edición (nivel 2).

\section{bibliotecas}




\section{Fundamentos y metodologías de investigación. Estudios métricos de información. 18 créditos. \\ Fundamentos, métodos y técnicas de investigación. Recogida y análisis de datos. Esta- dística descriptiva e inductiva. Elementos de análisis multivariante. Estudios métricos de información. Evaluación de la actividad científica. Estudios del uso de la información. \\ Estudios de usuarios. \\ Competencias: \\ Identificación, autentificación y evaluación de recursos de información (nivel 2). \\ Técnicas de diagnóstico y evaluación (nivel 2). \\ Esta materia tiene un componente transversal fundamental en la impartición de otras materias.}

\section{Practicum. 12 créditos.}

Conjunto integrado de prácticas en unidades de información.

Competencias:

En función del centro o servicio donde se realice, el Practicum puede tener una incidencia entre el nivel 2 y el 3 en diversas competencias de formación disciplinar y profesional.

\section{Contenidos Instrumentales obligatorios y Optativos}

Competencia en una segunda lengua, preferentemente el inglés (nivel 3), y aún en una tercera lengua.

Contenidos transversales relacionados con la sociedad de la información, cultura y sociedad, historia de las instituciones y de la administración pública, estructura y funcionamiento de las organizaciones públicas y privadas, o la filosofía e historia de la ciencia. Electivas.

Fuente: elaboración propia.

De acuerdo con el Libro Blanco, estos contenidos son en su mayoría de formación disciplinar y técnicas específicas de la información y la documentación, pero en algunas materias hay ciertos contenidos instrumentales que se han considerado troncales para asegurar su inclusión en los planes de estudio concretos. La propuesta de contenidos comunes obligatorios (troncalidad), pretende dar la formación básica para que un titulado de grado pueda trabajar en el ámbito de las bibliotecas, los archivos y los centros de documentación y como gestor de contenidos en cualquier tipo de organización, en un primer nivel de competencia profesional.

De esta manera, el titulado domina los fundamentos teóricos que están en la base de cada una de las competencias y tiene un "conocimiento de las prácticas", "puede manejar herramientas básicas y es capaz 
de efectuar ciertos trabajos técnicos", en cada una de las orientaciones profesionales que se han identificado. Las materias troncales han de asegurar la obtención de este primer nivel profesional. A partir de las materias obligatorias que determine cada universidad y de la optatividad el alumno podrá reforzar algunas de las competencias.

Excepto en el caso de las materias Representación y Recuperación de la Información y Gestión Técnica de Documentos de Archivo, en las que se han diseñado dos materias separadas para la organización y recuperación de los documentos bibliográficos y de los documentos de archivo, el resto de las materias se han concebido como comunes o armonizadas a todas las orientaciones profesionales. La recomendación del grupo de universidades que participó, los porcentajes de obligatoriedad/optatividad y la distribución correspondiente en horas de trabajo del alumno se presentan en la Tabla 5.

Es importante aclarar que existen diferencias en el manejo de los créditos en ambos países. Se entiende que el crédito comprende todas las actividades educativas requeridas: clases magistrales, clases prácticas, laboratorios, periodos de prácticas, ejercicios, lecturas, estudio, tutorías individuales y en grupo, preparación de exámenes y/o evaluaciones, etc. Por ello los análisis se harán en términos de porcentajes, ya que la medida del crédito europeo es 1 crédito $=25$-30 horas de trabajo del alumno, mientras que la medida del crédito colombiano es 1 crédito $=48$ horas de trabajo del alumno.

Tabla 5

Porcentaje de contenidos titulación española

\begin{tabular}{lccc}
\hline \multicolumn{1}{c}{ Libro Blanco } & Créditos & Porcentaje & Horas de trabajo del alumno \\
\hline Créditos de la titulación & 240 & $100 \%$ & $6000 / 7200$ \\
\hline Créditos troncales & 156 & $65 \%$ & $3900 / 4680$ \\
\hline Créditos obligatorios & $24-36$ & $10-15 \%$ (mínimo) & $\begin{array}{c}600 / 720-900 / 1080 \\
\text { (mínimo) }\end{array}$ \\
\hline Créditos optativos & $48-60$ & $20-25 \%$ (máximo) & $1200 / 1440$ - 1500/1800 (máximo) \\
\hline
\end{tabular}

Fuente: elaboración propia.

\section{bibliotecas}


Para analizar los contenidos propios de cada universidad sobre el total del título se revisaron los planes de estudio vigentes en 2012 y cada espacio académico con sus respectivos créditos fue ubicado de acuerdo con la propuesta de contenidos comunes obligatorios (troncalidad) del título de grado en Información y Documentación español. De esta manera, se logró establecer, para cada uno de los programas colombianos, el número de créditos, tanto en la troncalidad como en la obligatoriedad y optatividad, con respecto al total de créditos de cada programa, como se observa en la Tabla 6.

Tabla 6

Porcentaje de contenidos propios de cada universidad sobre el total del título

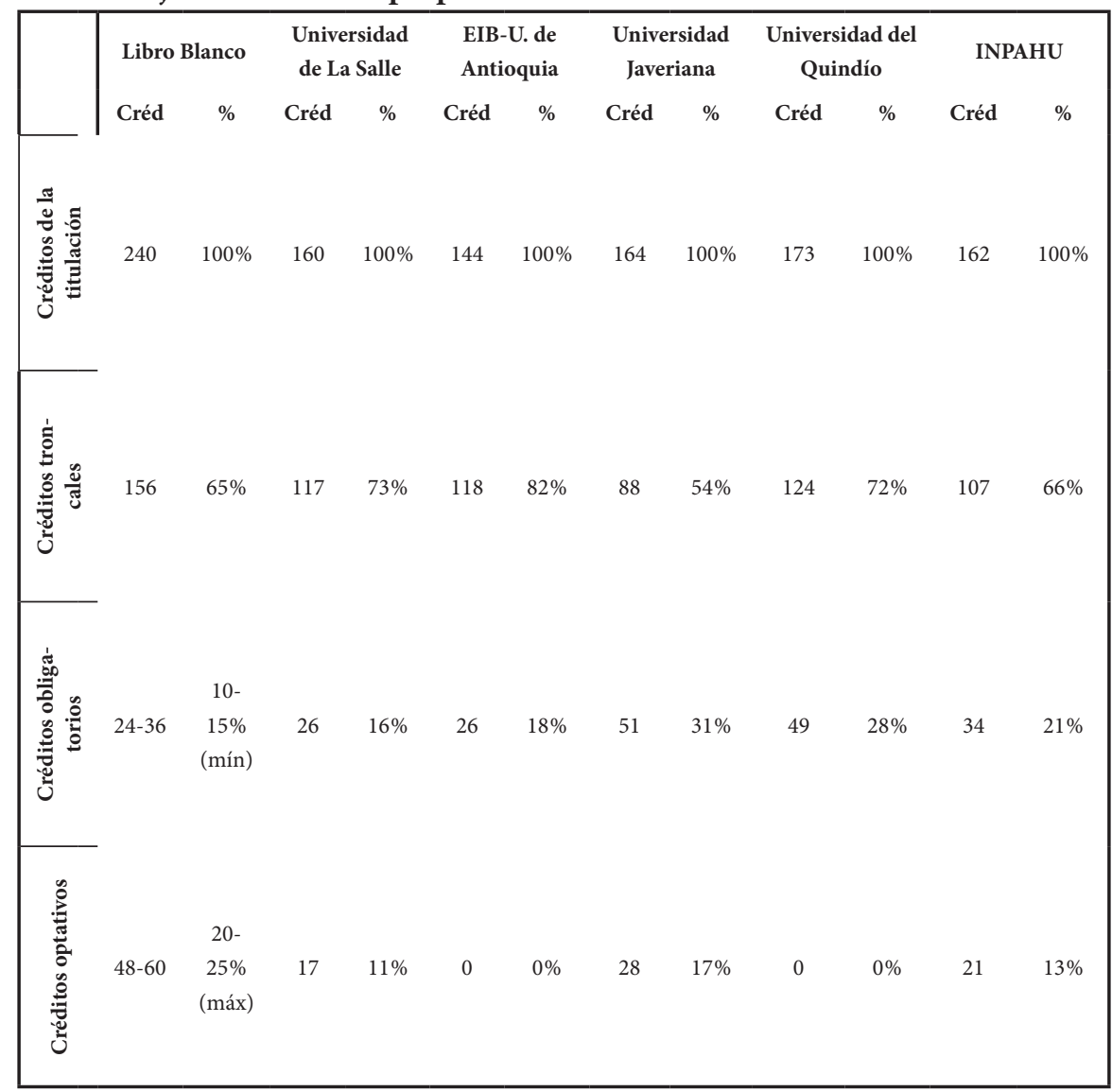

Fuente: elaboración propia. 


\section{Análisis de la información}

En un estudio más detallado por área, para cada universidad se puede observar lo siguiente:

\section{Tabla 7}

\section{Porcentaje de contenidos comunes obligatorios}

\begin{tabular}{ccccccc}
\hline Área & $\begin{array}{c}\text { Libro } \\
\text { Blanco }\end{array}$ & Salle & EIB & PUJ & UQ & INP \\
\hline $\begin{array}{c}\text { Documentos, unidades y siste- } \\
\text { mas de información }\end{array}$ & 5 & 9 & 10 & 9 & 17 & 6 \\
\hline $\begin{array}{c}\text { Planificación, organización } \\
\text { y evaluación de unidades de } \\
\text { información }\end{array}$ & 10 & 11 & 17 & 10 & 2 & 9 \\
\hline $\begin{array}{c}\text { Fuentes de información } \\
\text { de la información }\end{array}$ & 5 & 6 & 14 & 4 & 13 & 9 \\
\hline $\begin{array}{c}\text { Representación y recuperación } \\
\text { de archivo }\end{array}$ & 15 & 7 & 26 & 9 & 7 & 9 \\
\hline $\begin{array}{c}\text { Gestión técnica de documentos } \\
\text { decnologías de la información } \\
\text { y edición digital }\end{array}$ & 10 & 8 & 14 & 9 & 10 & 11 \\
\hline $\begin{array}{c}\text { Fundamentos y metodologías } \\
\text { de investigación. Estudios } \\
\text { métricos de información }\end{array}$ & 8 & 17 & 1 & 5 & 10 & 9 \\
\hline Practicum & 5 & 2 & 0 & 4 & 0 & 12 \\
\hline
\end{tabular}

Fuente: elaboración propia.

Documentos, unidades y sistemas de información: la recomendación es el 5\%. La mayor parte de las universidades colombianas superan este porcentaje, incluso lo doblan o triplican. Por ejemplo: la Universidad del Quindío (UQ) le otorga el mayor número de créditos a esta área (29), mientras el INPAHU (INP) solo le otorga (9). Por lo demás, las otras tres universidades tienen créditos muy parejos. Esta diferencia puede ocurrir porque el Programa de la Universidad del Quindío

\section{bibliotecas}


tiene espacios académicos especializados por cada tipo de unidad de información y fueron ubicados en esta categoría.

Planificación, organización y evaluación de unidades de información: contempla todo tipo de unidades, sean de la administración pública o de la empresa privada: bibliotecas, centros de documentación, archivos $\mathrm{u}$ otro tipo de organizaciones. Se sugiere el $10 \%$ y la mayor parte de las universidades se acerca; sin embargo, la Universidad de Antioquia (EIB) le otorga el 17\% de los créditos y la Universidad del Quindío solo el 2\%.

Fuentes de información: se recomienda el 5\% de los créditos. La Salle (ULS) y la Javeriana (PUJ) se acercan, mientras que la Universidad de Antioquia le otorga el 14\%, con 20 créditos, y la Universidad del Quindío el 13\%, con 23. La Universidad Javeriana le otorga el 4\%, es decir, 7 créditos.

Representación y recuperación de la información: se sugiere el 15\% de los créditos. La diferencia es considerable. La Universidad de Antioquia le otorga el $26 \%$, con 38 créditos y los demás programas entre el 7 y el $9 \%$, es decir, entre 11 y 15 créditos.

Gestión técnica de documentos de archivo: se recomienda el 8\%, que ninguna cumple. Tres universidades le dan el mismo peso, entre $12 \mathrm{y}$ 13\%, con 21 créditos Universidad del Quindío, Universidad de La Salle e INPAHU. La Universidad de Antioquia no le otorga créditos porque tiene programas profesionales independientes y la Universidad Javeriana le otorga el 4\%, con 6 créditos.

Tecnologías de la información y edición digital: se sugiere el 10\% de los créditos. En este caso todas son muy cercanas. Solo la Universidad de Antioquia le otorga el 14\%, con 20 créditos específicos, mientras que la Universidad de La Salle le otorga el 8\%, con 12 créditos.

Fundamentos y metodologías de investigación. Estudios métricos de información: $8 \%$ de los créditos indicados. La Universidad de La Salle le otorga el $17 \%$, con 28 créditos, mientras la Universidad de Antioquia solo le otorga el $1 \%$, con 2 créditos. 
Practicum: se indica el 5\%. Esta área hace parte de los planes de estudio de tres Universidades: Universidad Javeriana con el 4\% (6 créditos), La Salle con el 2\% (3 créditos) e INPAHU con el 1\% (2 créditos). Es importante aclarar que la Universidad de Antioquia y la Universidad del Quindío tienen esta área como modalidad de grado.

Es importante aclarar que no hay una sugerencia específica detallada para los contenidos instrumentales, ya que a partir de los créditos obligatorios y optativos, las universidades establecen, de acuerdo con sus prioridades y sus recursos, itinerarios que refuercen las orientaciones profesionales previstas en la definición del perfil profesional.

Contenidos instrumentales obligatorios: las universidades con mayor número de contenidos transversales son la Universidad del Quindío (49) y la Javeriana (51). Es importante mencionar que estas dos instituciones tienen en su plan de estudios créditos para una segunda lengua, mientras que las otras universidades lo establecen como requisito de grado. Vale la pena resaltar que en este grupo se incluyeron los espacios académicos exclusivos que tienen que ver con lectura, donde se observa que la Universidad de La Salle tiene dos, la Universidad de Antioquia dos, la Universidad Javeriana ninguno, la Universidad del Quindío nueve e INPAHU ninguno. Asimismo, en los espacios de contexto social se observa que el menor número de créditos lo tienen la Universidad de La Salle y la Universidad de Antioquia. Otro aspecto que mencionar en este campo, especialmente para la Universidad de La Salle y la Universidad Javeriana es la inclusión de espacios de contenido religioso, dado su carácter de universidades católicas.

Contenidos instrumentales optativos: hay una gran diferencia entre movilidad y flexibilidad de las titulaciones, ya que mientras la Universidad Javeriana tiene 28 créditos, la de Antioquia y la del Quindío no tienen ninguno.

De esta manera, al comparar los porcentajes sugeridos por el Libro Blanco en los contenidos troncales con respecto a las universidades colombianas, podemos decir que la Universidad Javeriana (PUJ) es la que más se acerca al modelo español, al tener los porcentajes más cer-

\section{bibliotecas}


canos en cinco de las ocho áreas (planificación, fuentes, tecnología, investigación y práctica), mientras que la Universidad de Antioquia (EIB) no tiene ningún área con porcentajes de créditos parecidos.

Es importante señalar que en el análisis es posible ver grandes diferencias en los modelos formativos español y colombiano, pues tanto en recuperación como en archivos ninguna coincide con lo propuesto (ver Tabla 8). Las áreas en las que se logró mayor acercamiento por número de universidades son planificación, tecnología e investigación.

\section{Tabla 8}

Análisis titulaciones colombianas por áreas

\begin{tabular}{|c|c|c|c|c|c|c|}
\hline Área & Breve descripción del contenido del área & Salle & EIB & PUJ & UQ & INP \\
\hline $\begin{array}{l}\text { Documentos, } \\
\text { unidades y } \\
\text { sistemas de } \\
\text { información }\end{array}$ & $\begin{array}{l}\text { Naturaleza de la información. Concepto y evolu- } \\
\text { ción de los documentos. Naturaleza y evolución } \\
\text { de los archivos, las bibliotecas y los centros y } \\
\text { servicios de documentación. Desarrollo histórico } \\
\text { y estado actual de la disciplina. Legislación y } \\
\text { políticas nacionales e internacionales. Aspectos } \\
\text { culturales, sociales, económicos y legales de la } \\
\text { producción y el uso de la información docu- } \\
\text { mental. Deontología y perfil del profesional de la } \\
\text { Información y Documentación. }\end{array}$ & 15 & 14 & 14 & 29 & 9 \\
\hline $\begin{array}{c}\text { Planificación, } \\
\text { organización } \\
\text { y evaluación } \\
\text { de unidades } \\
\text { de informa- } \\
\text { ción }\end{array}$ & $\begin{array}{l}\text { Planificación, organización y evaluación de } \\
\text { recursos, procesos, servicios y productos de } \\
\text { información. Desarrollo y mantenimiento de fon- } \\
\text { dos y colecciones. Programas de conservación y } \\
\text { preservación de documentos. Técnicas de gestión } \\
\text { de recursos humanos y financieros. Técnicas de } \\
\text { promoción: marketing y formación de usuarios. }\end{array}$ & 18 & 24 & 17 & 3 & 15 \\
\hline $\begin{array}{l}\text { Fuentes de } \\
\text { información }\end{array}$ & $\begin{array}{l}\text { Naturaleza y tipología de las fuentes de informa- } \\
\text { ción. Acceso y consulta de fuentes de informa- } \\
\text { ción electrónica. Análisis y evaluación de fuentes. } \\
\text { Producción, normalización y difusión de fuentes. }\end{array}$ & 10 & 20 & 7 & 23 & 14 \\
\hline $\begin{array}{l}\text { Represen- } \\
\text { tación y } \\
\text { recuperación } \\
\text { de la infor- } \\
\text { mación }\end{array}$ & $\begin{array}{l}\text { Catalogación descriptiva. Indización. Clasifi- } \\
\text { cación. Resumen. Desarrollo, mantenimiento } \\
\text { y evaluación de instrumentos de organización, } \\
\text { control y acceso. Elaboración, mantenimiento y } \\
\text { uso de lenguajes documentales. Técnicas de re- } \\
\text { cuperación de información. Sistemas avanzados } \\
\text { de recuperación de información. Evaluación de la } \\
\text { recuperación de información. }\end{array}$ & 11 & 38 & 15 & 12 & 14 \\
\hline
\end{tabular}




\begin{tabular}{|c|c|c|c|c|c|c|}
\hline $\begin{array}{l}\text { Gestión } \\
\text { técnica de } \\
\text { documentos } \\
\text { de archivo }\end{array}$ & $\begin{array}{l}\text { Organización de fondos de archivo. Identifica- } \\
\text { ción y valoración. Clasificación y descripción. } \\
\text { Acceso y difusión. Gestión del ciclo de vida de los } \\
\text { documentos administrativos. }\end{array}$ & 21 & 0 & 6 & 21 & 21 \\
\hline $\begin{array}{c}\text { Tecnolo- } \\
\text { gías de la } \\
\text { información } \\
\text { y edición } \\
\text { digital }\end{array}$ & $\begin{array}{l}\text { Arquitectura de ordenadores. Sistemas operati- } \\
\text { vos. Lenguajes de ordenadores. Sistemas y redes } \\
\text { de comunicación. Arquitectura de la informa- } \\
\text { ción. Programas de aplicación documental. } \\
\text { Fundamentos y diseño de bases de datos. Edición } \\
\text { de documentos digitales. Bibliotecas digitales. }\end{array}$ & 12 & 20 & 15 & 18 & 18 \\
\hline $\begin{array}{l}\text { Funda- } \\
\text { mentos y } \\
\text { metodologías } \\
\text { de inves- } \\
\text { tigación. } \\
\text { Estudios } \\
\text { métricos de } \\
\text { información }\end{array}$ & $\begin{array}{l}\text { Fundamentos, métodos y técnicas de investiga- } \\
\text { ción. Recogida y análisis de datos. Estadística } \\
\text { descriptiva e inductiva. Elementos de análisis } \\
\text { multivariante. Estudios métricos de información. } \\
\text { Evaluación de la actividad científica. Estudios del } \\
\text { uso de la información. Estudios de usuarios. }\end{array}$ & 27 & 2 & 8 & 18 & 14 \\
\hline Practicum & $\begin{array}{l}\text { Conjunto integrado de prácticas en unidades de } \\
\text { información. }\end{array}$ & 3 & 0 & 6 & 0 & 2 \\
\hline $\begin{array}{l}\text { Contenidos } \\
\text { instrumen- } \\
\text { tales obliga- } \\
\text { torios }\end{array}$ & $\begin{array}{l}\text { Competencia en una segunda lengua, preferen- } \\
\text { temente el inglés (nivel } 3 \text { ), y aún en una tercera } \\
\text { lengua. Contenidos transversales relacionados } \\
\text { con la sociedad de la información, cultura y } \\
\text { sociedad, historia de las instituciones y de la } \\
\text { administración pública, estructura y funciona- } \\
\text { miento de las organizaciones públicas y privadas, } \\
\text { o la filosofía e historia de la ciencia. }\end{array}$ & 26 & 26 & 51 & 49 & 34 \\
\hline $\begin{array}{l}\text { Contenidos } \\
\text { instrumenta- } \\
\text { les optativos }\end{array}$ & Electivas & 17 & 0 & 28 & 0 & 21 \\
\hline
\end{tabular}

Fuente: elaboración propia.

Así, con respecto a las recomendaciones realizadas para cada titulación, solamente la Universidad Javeriana tiene créditos troncales inferiores (56\%) en relación con los sugeridos (65\%), en tanto que tres: la Universidad de La Salle, la Universidad de Antioquia y la Universidad del Quindío tienen valores mayores al 70\%. Lo cual quiere decir que en el desarrollo de los planes de estudio colombianos se le da un mayor énfasis a los contenidos propios de la disciplina.

En cuanto a los créditos obligatorios, todas las universidades cumplen con las sugerencias ya que mantienen entre el 16\% y el $31 \%$ de los cré-

\section{bibliotecas}


ditos en esta área, lo cual demuestra el interés de cada institución por otorgar su valor agregado diferente de formación.

En cuanto a los créditos optativos, estos oscilan entre 11 y $17 \%$, aunque la Universidad de Antioquia y la del Quindío no cuentan con esta modalidad. De esta manera, se destaca el interés de la Universidad Javeriana por dar mayor selectividad a sus estudiantes, con el 17\% de los créditos

De esta manera y como punto de partida para comprender las rutas de formación en Colombia, de acuerdo con la titulación en Información y Documentación española, las universidades colombianas se pueden analizar de la siguiente manera:

- Universidad de La Salle: el mayor énfasis de su plan de estudios está en la investigación y en los contenidos transversales relacionados con la disciplina, seguida por la formación en archivos.

- Universidad de Antioquia: su mayor fortaleza es la representación y recuperación de la información, seguida de los contenidos transversales relacionados con la disciplina y posteriormente la planificación, organización y evaluación de unidades de información.

- Pontificia Universidad Javeriana: su valor agregado son los contenidos transversales relacionados con la disciplina, luego las electivas y la planificación, organización y evaluación de unidades de información.

- Universidad del Quindío: se destacan los contenidos transversales relacionados con la disciplina, documentos, unidades y sistemas de información y fuentes de información.

- INPAHU: enfatiza en los contenidos transversales relacionados con la disciplina, las electivas y los contenidos de archivos.

\section{Conclusiones}

Cada escuela diseña currículos con los cuales, además de tratar de abarcar todos los conocimientos del campo y responder a los requerimientos 
de la sociedad y los empleadores, asigna un sello diferenciador o valor agregado a la formación que define el perfil profesional de los egresados de cada institución educativa. En este sentido, Rojas (2010) resalta de la formación profesional y el conjunto de procesos sociales de preparación y conformación del sujeto, referido a fines precisos para un posterior desempeño en el ámbito laboral. Este se basa en el abordaje, dominio y manejo de un cuerpo de conocimientos teóricos e instrumentales (saberes diferenciados) sobre determinado campo del saber, ciencia, quehacer o disciplina, vinculando la formación profesional a dos referentes básicos: el terreno del conocimiento y el ámbito de la realidad, cuyo marco educativo es de carácter institucional, regula las actividades consideradas como necesarias y pertinentes y avala la oferta de formación con la expedición de certificados y títulos profesionales.

Los procesos formativos tienen un amplio contenido disciplinar que se refleja en el mayor número de créditos en esta área, pero no se destaca por ningún área específica, con lo cual no hay una competencia significativa que los distinga; es decir, se han conformado planes de estudios completos con las condiciones básicas que debe tener la formación profesional de un bibliotecólogo, y esto muestra el claro compromiso de las universidades de contar con profesionales idóneos y la calidad de la educación bibliotecológica colombiana.

En el contexto colombiano es indispensable considerar la diferencia existente entre archivística y bibliotecología, pues no solo tienen formación independiente, sino que cuentan con leyes diferentes en cuanto a su ejercicio profesional. Este hecho impacta notablemente en la profesión. Es importante mencionar los cambios significativos que ha conllevado en los planes de estudio, pues esto ya se ve reflejado en una posición mucho más clara sobre el tema y el interés que demanda tanto para las instituciones como para la sociedad colombiana. Evidentemente hay una problemática que se refleja y es la falta de un tronco común de formación y el gran interés de todas las universidades por que exista un factor predominante que las diferencie unas de otras a través de los contenidos transversales.

\section{bibliotecas}




\section{Recomendaciones}

El momento que está viviendo la bibliotecología, en cuanto al impacto de una sociedad en permanente cambio, pone a las escuelas de bibliotecología y ciencia de la información ante la necesidad de renovar permanentemente sus currículos, permitiendo preparar profesionales que respondan a las exigencias de la sociedad, ampliando los campos de acción de los bibliotecólogos y fortaleciendo el posicionamiento de la profesión, a fin de que se permita un mayor cubrimiento ante las escasas solicitudes de ingreso que se reciben. Esto nos pone a reconsiderar algunos de las recomendaciones del Informe Williamson.

La formación debe partir de un tronco disciplinar, proceso que ha logrado unificarse. Vale la pena en este sentido destacar lo logrado en España con la titulación en Información y Documentación, los avances alcanzados por Mercosur, o la misma apuesta de la American Library Association con sus estándares de calidad para las maestrías. Los exámenes de calidad de la educación superior específicos son una nueva obligación que deben asumir los futuros profesionales y obligarán a las escuelas a ponerse de acuerdo sobre las competencias y conocimientos mínimos del bibliotecólogo colombiano. Por ello no se puede continuar con programas dispersos, con titulaciones diversas y desarticuladas. Además, se debe pensar en:

- La bibliotecología enseñada como disciplina teórica, superándose la formación técnica.

- El desarrollo y énfasis en la investigación y el contexto social.

- El compromiso para innovar la enseñanza y el contenido de los programas de estudio, así como los métodos de evaluación del desempeño de los estudiantes, pero al mismo tiempo establecer criterios más apropiados para el ingreso a la profesión.

- Incluir nuevas prácticas que posibiliten su trabajo en equipos multidisciplinarios. 
- Mejorar la preparación del personal docente para reconfigurar las metodologías de enseñanza, renovar los perfiles de vinculación a las escuelas y entender que los docentes tienen la obligatoriedad de formarse pedagógicamente.

- La división del trabajo técnico, tecnológico y profesional, lo cual requiere la formación de profesionales en todos los niveles e implica un desafío para desarrollar currículos que se adapten a las necesidades de cada grupo.

- Incluir en las mallas curriculares el proceso de profesionalización, su desarrollo, sus problemáticas y el papel de cada uno de ellos, aportando a la construcción de la identidad.

- Desarrollar la capacidad crítica y reflexiva del bibliotecólogo.

\section{Referencias}

Agencia Nacional de Evaluación de la Calidad y Acreditación (ANECA) 2004. Libro Blanco: Título de grado en información y documentación. Recuperado de http://www.aneca.es/var/media/150424/libroblanco_jun05_documentacion.pdf

Linares, R. 2004. La Bibliotecología y sus orígenes. Ciencias de la Información. 35, (3). Recuperado de http://www.cinfo.cu/Userfiles/ file/Cinfo/cinfo2004/diciembre_35/Bibliotecologia.pdf

Rios Ortega, Jaime (2010). Perfil de competencias en bibliotecología para la región latinoamericana: exordio a su elaboración. En: Lina Escalona de Ríos, las competencias en el perfil bibliotecológico en América Latina. Recuperado de http://libros.metabiblioteca.org/bitstream/001/218/8/competencias_perfil.pdf

Rodríguez, J. (2001). Formación humanística del bibliotecólogo: hacia su recuperación. Ciudad de México : Universidad Autónoma de México.

Rojas, M. (2010). Usos y apropiaciones de las tecnologías de la información y comunicación en la formación del comunicador social,

\section{bibliotecas}


caso: Universidad Veracruzana. Recuperado de www.eumed. net/libros/2010a/634/

Shera, Jesse H.(1990). Los fundamentos de la educación bibliotecológica. México: UNAM, Centro Universitario de Investigaciones Bibliotecológicas.

Unesco (1987). Armonización de la capacitación en materia de Biblioteconomía, Ciencias de la Información y Archivística. PGI-87/ WS/2. Paris: UNESCO, Programa General de Información.

Vallejo Sierra, Ruth Elena (2010). La formación profesional de bibliotecólogos y archivistas: integrada, con énfasis o independiente. Recuperado de http://www.ifla.org/past-wlic/2011/110-sierra-es.pdf

\section{Notas de la autora}

* Ruth Helena Vallejo Sierra: Doctora en Documentación. Reside en Bogotá, Colombia. El artículo es derivado de la investigación doctoral La bibliotecología como profesión en Colombia, presentada en la Universidad de Salamanca, España. Correo electrónico: Rhvs2007@gmail.com 